\title{
DIFERENCIAS SEXUALES EN LOS RASGOS DE PERSONALIDAD INSTRUMENTALES Y EXPRESIVOS DE ADOLESCENTES ESPAÑOLES
}

\author{
María de la Paz Toldos-Romero \\ Tecnológico de Monterrey, Campus Guadalajara - México. \\ José Luis Rojas-Solís \\ Benemérita Universidad Autónoma de Puebla - México \\ Javier Martín-Babarro \\ Facultad de Psicología, Universidad Complutense de Madrid \\ https://doi.org/10.17060/ijodaep.2017.n1.v2.266
}

Fecha de Recepción: 28 Marzo 2017

Fecha de Admisión: 1 Abril 2017

\section{RESUMEN}

Investigaciones señalan que la gente suele creer que hombres y mujeres tienen rasgos de personalidad diferentes caracterizando a las mujeres con rasgos "expresivos" y a los varones como "instrumentales". Debido a lo controvertido del tema la presente investigación examina las diferencias sexuales en los rasgos instrumentales y expresivos de 634 adolescentes, con edades comprendidas entre 14 y 18 años, quienes cumplimentaron el Cuestionario de Atributos Personales (Spence, Helmreich y Stapp, 1974). Los resultados muestran diferencias entre sexos en la autoidentificación con los valores tradicionales habitualmente atribuidos al otro sexo al igual que un cambio en los estereotipos de rol sexual, siendo la superación del estereotipo sexista más clara para las chicas quienes se identifican con más rasgos instrumentales.

Palabras clave: Instrumentalidad; Expresividad; Diferencias sexuales; Estereotipos de rol sexual

\section{ABSTRACT}

\section{Sex differences in Spaniard adolescents' instrumental and expressive traits}

Extensive research has shown that people believe that men and women have different personality traits characterizing women with "expressive" traits and men with "instrumental" traits. Because of the controversial nature of this topic, this research examines sex differences in the expressive and instrumental traits of 634 adolescents aged between 14 to 18 years, who answered the Personal Attributes Questionnaire (Spence, Helmreich \& Stapp, 1974). The results show sex differences in self-identification with traditional values usually attributed to the other sex, also a change in the sex role stereotypes values, being the overcoming of the sexist stereotype clearer for girls who identified themselves with more instrumental traits.

Key words: Instrumentality; Expressiveness; Sex differences; Sexual role stereotypes 


\section{DIFERENCIAS SEXUALES EN LOS RASGOS DE PERSONALIDAD INSTRUMENTALES Y EXPRESIVOS DE ADOLESCENTES ESPAÑOLES}

\section{ANTECEDENTES}

Las primeras aproximaciones que fundamentan el estudio del género se realizaron a través de la operacionalización de los denominados rasgos de masculinidad y feminidad que caracterizan a los varones como independientes y dominantes en el espacio público y a las mujeres como sensibles y relegadas al ámbito privado/doméstico (Rodríguez, Lameiras, Magalhaes y Carrera, 2010). No obstante, estos conceptos han evolucionado desde un modelo clásico de unidimensionalidad hasta los modelos multidimensionales. Entre estos modelos teóricos sobre la identidad de género destaca la teoría del Esquema de Género de Bem $(1981,1982)$ donde los sujetos tipificados sexualmente, varones masculinos y mujeres femeninas, son esquemáticos a la hora de procesar la información sobre el género. Mientras que otras propuestas como el modelo del Auto-Esquema de Markus, Crane, Bernstein y Siladi (1982), o la perspectiva de Spence (1983) proponen que las actitudes, atributos y comportamientos referidos al género son multidimensionales y que a partir de la categorización de los sujetos tan sólo puede hacerse referencia a una de las dimensiones del género, es decir, la dimensión instrumentalidad-expresividad (Vergara y Páez, 1993).

Por otro lado, un gran número de investigaciones han demostrado que la gente cree que los varones y las mujeres tienen rasgos de personalidad diferentes (Bem, 1974; Rosenkrantz, Vogel, Bee, Broverman, y Broverman, 1968; Spence, Helmreich, y Stapp, 1975) caracterizando a las mujeres con rasgos "expresivos" que expresan calidez, preocupación por los otros, empatía, debilidad, y dependencia, entre otros, y a los varones con rasgos "instrumentales" que expresan agresividad, competitividad, dureza, insensibilidad, etc. También se ha señalado que estos rasgos son considerados no sólo típicos para ambos sexos sino también deseables de manera que ambos sexos se sienten presionados a ajustarse a ellos y creen que deben manifestar las características esperadas de su sexo para tener una apropiada identidad de género (Gerber, 1989).

Es precisamente en este proceso de "cumplir" con las expectativas culturales que varones y mujeres siguen manteniendo las relaciones tradicionales entre los sexos, de modo que los roles de ambos interactúan en su vida diaria manteniendo o reforzando los estereotipos sobre el género. Por ello es importante estudiar a fondo cómo estas expectativas definidas socioculturalmente como propias de un sexo u otro afectan a varones y mujeres en sus representaciones de sí mismos y más específicamente en los jóvenes, etapa crítica donde éstos construyen su identidad y se intensifican las actitudes y conductas de género (Siverio y García, 2007).

Por otro lado en gran parte de los estudios sobre el desarrollo de la identidad en la adolescencia, los chicos se muestran ligeramente más avanzados en los aspectos que tienen que ver con la identidad intrapersonal (ocupación, autonomía, individuación, etc.), mientras que las chicas lo están en los aspectos más relacionados con la identidad personal, relaciones, familia, etc. (Harter, 1990). Y aunque en los últimos años en ámbitos educativos se ha trabajado desde la no discriminación por razón de sexo y la flexibilidad de roles de género e igualdad de oportunidades entre chicos y chicas, es evidente que ambos sexos siguen siendo socializados con normas y expectativas distintas definidas socioculturalmente como propias de un sexo u otro en lo que concierte a la expresión de los sentimientos, entre otros.

En este sentido algunas investigaciones (Spence, 1993; Spence y Buckner, 2000) encuentran que tanto las chicas como los chicos tienden a evaluarse con las características congruentes con su propio género. De manera que los chicos se perciben con rasgos instrumentales propios y deseables de su sexo, mientras que las chicas responden a elementos expresivos deseables y característicos de las mujeres. Mediante la representación de las expectativas de rol los chicos deben ser asertivos y las chicas, estar orientadas hacia las relaciones interpersonales. 


\section{PLANTEAMIENTO Y OBJETIVOS}

Al igual que Spence y Buckner (2000) sugieren, entendemos que algunos elementos o rasgos propios de un sexo u otro puedan haberse modificado en la percepción que chicos y chicas tienen de sí mismos debido a los cambios sociales, culturales y ambientales (Twenge, 1997) que han tenido lugar en los últimos tiempos afectando a las diferencias en cómo chicos y chicas se ven a sí mismos y en sus creencias estereotipadas con sus iguales. Por lo anterior el objetivo de la presente investigación es evaluar los estereotipos de género de mujeres y hombres adolescentes, y comprobar si existen diferencias significativas en los rasgos instrumentales y expresivos en función del sexo, curso y nivel socioeconómico de la zona donde se ubica su centro escolar. El interés recae en evaluar si ha cambiado la auto-percepción que chicos y chicas tienen de sí mismos debido a los cambios sociales que permiten una mayor flexibilidad en los roles de género. Igualmente se pretende establecer la dimensionalidad del Cuestionario de Atributos Personales de Spence, Helmreich y Stapp (1974) de masculinidad y feminidad a través de rasgos de personalidad instrumentales y expresivos.

\section{MÉTODO}

\section{Participantes}

Los participantes de esta investigación pertenecen a diferentes Centros Educativos de Educación Secundaria Obligatoria y Bachillerato de la Comunidad de Madrid. El tamaño total de la muestra es de 634 sujetos, de los cuales 307 son chicos y 326 son chicas, con edades comprendidas entre 14 y 18 años. La edad media de los chicos es $15.51(D T=.97)$ y la de las chicas es $15.56(D T=1.05)$.

\section{Instrumento de medida}

Se utilizó el cuestionario de Atributos Personales (PAQ; Spence et al., 1974) en su formato abreviado, que evalúa la masculinidad y la feminidad a través de rasgos de personalidad instrumentales y expresivos. Esta escala consta de 24 ítems de descripción personal bipolar que implica rasgos de personalidad y motivación, valorados en una escala Likert de cinco puntos. Los coeficientes de consistencia interna para esta escala han alcanzado valores altos en otras investigaciones, como la realizada por Helmreich, Spence y Wilhelm (1981) quienes hallaron coeficientes de .75 para la escala de masculinidad, .91 para la de feminidad y .78 para la escala de masculinidad-feminidad.

\section{Procedimiento}

La selección de los centros escolares fue incidental y considerando dos criterios: 1) tipo de educación (concertado-religioso, público no concertado-religioso) y 2) estatus socioeconómico de la zona (medio-bajo, medio-alto). Del total de escuelas se seleccionaron cuatro: dos centros escolares público y concertado religioso de nivel medio-bajo y otros dos público y concertado religioso de nivel medio-alto. La selección de la muestra se llevó a cabo aleatoriamente dentro de cada centro, los dos sexos estaban equilibrados en todos los niveles de edad al igual que existió bastante correspondencia entre las distribuciones de sexos por centros y de edades por cursos escolares. La evaluación se realizó dentro de las horas de clases y el llenado de los cuestionarios requirió una duración aproximada de 20 minutos.

\section{Análisis de datos}

En primer lugar se estableció la dimensionalidad de la escala PAQ por medio de la factorización de la matriz de correlaciones entre sus elementos utilizándose el procedimiento de Componentes Principales, seguido de la rotación Varimax. Acto seguido se calculó la fiabilidad por medio del 


\section{DIFERENCIAS SEXUALES EN LOS RASGOS DE PERSONALIDAD INSTRUMENTALES Y EXPRESIVOS DE ADOLESCENTES ESPAÑOLES}

Coeficiente Alpha de Cronbach. En segundo lugar se realizaron análisis descriptivos de todos los elementos de los cuestionarios y contrastes de diferencias de medias para muestras independientes con el estadístico $t$ de Student para hallar las diferencias entre sexos y cursos. Los contrastes se realizaron con el procedimiento de varianzas iguales en los casos en que el test de Levene soportó el supuesto, y con varianzas desiguales y grados de libertad calculados cuando las varianzas resultaron significativamente diferentes. Se calculó el coeficiente Eta como una medida del tamaño del efecto del género en la variabilidad de las respuestas.

Por último, para el examen de las diferencias en el tipo de educación, se llevaron a cabo Análisis de Varianza (ANOVA) de un factor con cuatro niveles. En los casos en los que se apreció alguna diferencia significativa las pruebas a posteriori fueron realizadas con el contraste de Games-Howell.

Los análisis estadísticos fueron realizados con el paquete estadístico SPSS, versión 18.0.

\section{RESULTADOS}

\section{Análisis de la dimensionalidad y fiabilidad de la escala}

El conjunto de los 2 factores explicó el $31.7 \%$ de la varianza total. Los factores de la solución rotada explicaron los siguientes porcentajes de la varianza total: Factor 1: 17.97\%; Factor 2: $13.73 \%$. Un examen de las saturaciones, permite establecer los elementos que definen cada factor así como la naturaleza de los mismos (ver Tabla 1).

Factor 1. Expresividad. Formado por 14 elementos donde se incluyen afirmaciones que hacen referencia a valores de sensibilidad emocional, calidez e inseguridad (Ítems: emocional, muy sumiso/a, excitable en una crisis importante, nada competitivo/a, muy hogareño/a, nada indiferente a la aprobación de los demás, sus sentimientos son heridos con facilidad, no puede tomar decisiones fácilmente, se da por vencido/a fácilmente, siempre llora, nada seguro/a de sí mismo/a, se siente muy inferior, con mucha necesidad de seguridad y se desmorona bajo presiones).

Factor 2. Instrumentalidad. Formado por 10 elementos que incluyen afirmaciones que hacen referencia a la agresividad-dureza e individualismo, también trata de adjetivos que incluyen la pasividad e inseguridad (Ítems: agresivo/a, nada independiente, muy pasivo/a, incapaz de dedicarse totalmente a los otros, muy duro/a, nada servicial con los demás, nada amable, nada consciente de los sentimientos de los demás, nada comprensivo/a con los demás y muy frío/a en las relaciones con los demás).

Se calculó la fiabilidad mediante el procedimiento de consistencia interna de cada una de las subescalas derivadas mediante el análisis factorial por medio del coeficiente Alpha de Cronbach. Todos los elementos presentan coeficientes de discriminación que superan el valor de .40, encontrándose un coeficiente alpha de .70 para el total de la escala y de .77 para el factor 1 y de .70 para el factor 2. En resumen, el valor del coeficiente Alpha para cada uno de los factores es alto. 
Tabla 1.

Matriz de configuración de factores del cuestionario

\begin{tabular}{|c|c|c|}
\hline \multirow[t]{2}{*}{ Ítems } & \multicolumn{2}{|c|}{ FACTORES } \\
\hline & 1 & 2 \\
\hline 1. Nada agresivo/a & .102 & -.199 \\
\hline 2. Nada independiente & & .126 \\
\hline 3. Nada emocional & -.506 & .443 \\
\hline 4. Muy sumiso/a & .276 & \\
\hline 5. Nada excitable en una crisis importante & -.263 & \\
\hline 6. Muy pasivo/a & .188 & .342 \\
\hline 7. Incapaz de dedicarse totalmente a los otros & -.174 & .654 \\
\hline 8. Muy duro/a-brutal & -.308 & .458 \\
\hline 9. Nada servicial con los demás & & .680 \\
\hline 10. Nada competitivo/a & .448 & \\
\hline 11. Muy hogareño/a & .237 & \\
\hline 12. Nada amble & .142 & .580 \\
\hline 13. Indiferente a la aprobación de los demás & -.398 & \\
\hline 14. Mis sentimientos son heridos con facilidad & .681 & .258 \\
\hline 15. Nada consciente de los sentimientos de los demás & -.175 & .619 \\
\hline 16. Puedes tomar decisiones fácilmente & -.586 & \\
\hline 17. Te das por vencido/a fácilmente & .578 & .328 \\
\hline 18. Nunca lloras & -.637 & .400 \\
\hline 19. Nada seguro/a de ti mismo/a & .706 & .313 \\
\hline 20. Te sientes muy inferior & .561 & \\
\hline 21. Nada comprensivo/a con los demás & & .764 \\
\hline 22. Muy frío/a en las relaciones con los demás & & 608 \\
\hline 23. Muy poca necesidad de seguridad & -.257 & .155 \\
\hline 24. Te desmoronas bajo presiones & .697 & \\
\hline
\end{tabular}

Nota: Para claridad en la interpretación se han omitido las saturaciones inferiores a .25 


\section{DIFERENCIAS SEXUALES EN LOS RASGOS DE PERSONALIDAD INSTRUMENTALES Y EXPRESIVOS DE ADOLESCENTES ESPAÑOLES}

\section{Diferencias en función del sexo}

Para explorar las diferencias en función del sexo en la identificación con los valores expresivos e instrumentales, se llevaron a cabo contrastes $t$ de Student para muestras independientes.

Como puede observarse en la Tabla 2, Ios y las adolescentes difieren significativamente $(p<.01)$ en las puntuaciones de los 2 factores. En el factor 1 que mide expresividad, tradicionalmente asociado con estereotipos femeninos, los chicos puntúan más alto. Para el factor 2 que mide instrumentalidad, tradicionalmente asociado con estereotipos masculinos, las chicas puntúan más alto.

Tabla 2.

Medias y desviaciones típicas de las puntuaciones en los factores según el sexo

\begin{tabular}{cccccccc}
\hline Factores PAQ & Sexo & M & DT & $\begin{array}{c}\text { Error } \\
\text { típico } \\
\text { de la } \\
\text { media }\end{array}$ & t (gl) & Eta \\
\hline \multirow{2}{*}{ Expresividad } & Mujer & 2.77 & .51 & .39 & $-11.937^{* *}$ & .429 \\
& Varón & 3.25 & .48 & .39 & $(630)$ & \\
Instrumentalidad & Mujer & 3.98 & .41 & .23 & $9.665^{* *}$ & .359 \\
& Varón & 3.64 & .47 & .27 & $(632)$ & \\
\hline $\mathrm{p}<01$
\end{tabular}

$* \mathrm{p}<.05 ; * * \overline{\mathrm{p}<.01}$

Para comprobar las diferencias en función del sexo para cada uno de los elementos 0 items que componen el factor 1 y 2 , se llevaron a cabo contrastes $t$ de Student. Para el factor 1 que mide expresividad, los adolescentes puntúan más alto en diez de los 14 elementos que componen la subescala. Es decir, que los chicos, en comparación con las chicas, se consideran: nada competitivos, nada seguros, se sienten inferiores, se desmoronan bajo presiones, muy emocionales, necesitados de la aprobación de los demás, sus sentimientos son heridos con facilidad, tienen dificultad para tomar decisiones, lloran fácilmente y necesitan seguridad. Para los otros cuatro ítems que componente el factor (sumisos, excitables en una crisis importante, hogareños y se dan por vencidos fácilmente) no se hallaron diferencias significativas entre chicos y chicas.

Para el factor 2 que mide instrumentalidad, se encontraron diferencias significativas en siete de los diez elementos que componen el factor. En este factor las chicas puntúan más alto que los chicos en: incapaz de dedicarse a los otros, duras, nada serviciales, nada conscientes de los sentimientos de los demás, nada comprensivas con los otros, agresivas y frías en las relaciones con los demás. Para los tres elementos restantes (nada independiente, pasivo/a y nada amable) no se encontraron diferencias significativas entre los y las adolescentes.

\section{Diferencias en función del curso y del nivel socioeconómico de la zona}

Para evidenciar las posibles diferencias en función del curso escolar se dividió la muestra en dos grupos, los que cursan secundaria y los que cursan bachillerato. Así, mediante la comparación de medias para cada curso ( $t$ de Student) se encontró que los que cursan secundaria y bachillerato difieren significativamente $(p<0.01)$ en las puntuaciones del factor 1 , expresividad $\left(F_{630}=2.566\right.$, $p<.000)$. Es decir, en el factor 1 , tradicionalmente asociado con estereotipos femeninos, los alumnos de secundaria $(\mathrm{M}=3.06$, $\mathrm{DT}=.52)$ puntúan más alto que los de bachillerato $(\mathrm{M}=2.87$, $\mathrm{DT}=$ 
.59). Mientras que para el factor 2 , tradicionalmente asociado con estereotipos masculinos, no hay diferencias entre los dos grupos $\left(\mathrm{F}_{632}=.001, p=-.720\right)$.

En cuanto al examen de las diferencias en el tipo de educación y en la edad, se llevaron a cabo ANOVA's de un factor con cuatro niveles. En cuanto a las diferencias en función del nivel socioeconómico de la zona se obtuvieron diferencias estadísticamente significativas únicamente para el factor 1, expresividad, $\left(\mathrm{F}_{631}=2.678, p=.046\right)$, de manera que los alumnos/as del centro escolar religioso-concertado de nivel medio-bajo puntúan más alto en este factor que los alumnos/as del centro público de nivel medio-bajo. En cuanto a las diferencias por edad, se obtienen diferencias estadísticamente significativas en el factor 1 , expresividad $\left(\mathrm{F}_{631}=4.950, p=.02\right)$, de modo que los y las adolescentes de 15 años puntúan más en este factor que los de 14, 16 y 17 años.

\section{DISCUSIÓN Y CONCLUSIONES}

A través de la escala utilizada en esta investigación se pudo evaluar cómo varían los rasgos expresivos e instrumentales tradicionalmente atribuidos a varones y mujeres. Así, el conjunto de los resultados obtenidos apoyan la idea de que los cambios sociales parecen haber dado lugar a un cambio en la percepción que tienen las chicas sobre sus rasgos instrumentales aproximándose más a los de los chicos.

Se encontraron diferencias entre chicos y chicas en la identificación con los valores tradicionalmente atribuidos al otro sexo, según los cuales la superación del sexismo es más clara en las adolescentes que entre los adolescentes, ya que las chicas se ven a sí mismas con rasgos o características más instrumentales. Por otro lado, se esperaba encontrar que los chicos se identificaran con los valores tradicionalmente instrumentales, sin embargo, se identificaron con los valores expresivos tradicionalmente asociados a las mujeres.

Resultados que coinciden parcialmente con lo hallado por Díaz-Aguado, Martínez, Martín y Toldos (2001) quienes también encontraron cambios importantes en la superación del estereotipo femenino. En contra parte otros estudios (Finn, 1986; Klein, Campbell, Soler y Ghez, 1997; Strauss, Kaufman y Moore, 1997) sugieren que aunque en los últimos años se ha producido un avance considerable en la superación del sexismo por parte de chicos y chicas, dicha superación dista todavía mucho de ser total, especialmente en los varones, existiendo una mayor tendencia en las chicas al cambio en la identidad de género (Pastorino, Dunham, Kidwell, Bacho y Lamborn, 1997).

Por tanto, el conjunto de valores y problemas tradicionalmente asociado al estereotipo femenino de expresividad-comunalidad parece haberse modificado en la imagen real que tienen las adolescentes de sí mismas, al igual que el conjunto de valores tradicionalmente asociados al estereotipo masculino de instrumentalidad-agencia. Todo ello podría explicarse considerando los cambios culturales, las expectativas que actualmente se tienen desde la niñez, las contradicciones en el proceso de socialización de los roles de género, el cambio de valores y las influencias del ambiente cultural y económico de nuestro tiempo. Los cambios ocurridos en las últimas décadas han provocado cambios profundos en los roles de género, entre ellos la menor restricción en la socialización de los niños/as, el mayor número de madres que trabajan fuera de casa, las oportunidades educativas para las mujeres, la reducción del tiempo dedicado al embarazo y maternidad, la disminución del tamaño de la familia, el incremento y énfasis de la sociedad por los derechos de la mujer, o el trabajo de las mujeres y los roles profesionales, sobre todo instrumentales, que han adquirido las mujeres para destacar en los roles profesionales y académicos, entre otros. Por lo que no es de extrañar la visión de la nueva mujer como masculina, ambiciosa, orientada hacia la carrera, asertiva, lógica, etc. (Toldos, 2002).

Todos estos avances son sólo algunos ejemplos del cambio social que ciertamente pueden vivirse en la actualidad, sin embargo probablemente han incidido menos al interior de la dinámica fami- 


\section{DIFERENCIAS SEXUALES EN LOS RASGOS DE PERSONALIDAD INSTRUMENTALES Y EXPRESIVOS DE ADOLESCENTES ESPAÑOLES}

liar (siempre más tradicional) y quizás por ello en este estudio se haya encontrado que los jóvenes se ven a sí mismos/as con roles menos tradicionales. Así las adolescentes estarían copiando los roles masculinos o instrumentales, es posible que estén copiándolos y que sigan madurando con estos roles, o bien sólo estén utilizando las conductas instrumentales asociadas al estereotipo masculino como una forma de afrontar la incertidumbre que se vive en la adolescencia.

Por lo anterior se deduce la importancia de la detección en el contexto escolar de estos estereotipos en aras de fomentar la igualdad de género, prevenir prejuicios basados en el sexo-género y la discriminación de uno u otro por medio de programas de educación para la igualdad y un currículo no sexista.

Por último, sería pertinente que futuras investigaciones implementasen diseños de naturaleza longitudinal que evalúen los rasgos instrumentales y expresivos desde la etapa de la adolescencia hasta la edad adulta para identificar el grado de su preservación o mutación.

Asimismo la información obtenida se podría enriquecer con la inclusión de otras variables como la visión que chicos y chicas tienen de sus iguales del otro sexo o incluso de varones y mujeres de edad adulta para identificar el nivel de sexismo interiorizado y estereotipos hacia mujeres y varones. Finalmente, sería importante realizar estudios transculturales y evaluar si los programas de igualdad y no discriminación en las escuelas están influyendo, o no, en la percepción que chicos y chicas tienen de sí mismos.

\section{REFERENCIAS}

Bem, S. L. (1974). The measure of psychological androgyny. Journal of Consulting and Clinical Psychology, 42(2), 155-162. doi:10.1037/h0036215

Bem, S. L. (1981). Gender schema theory: A cognitive account of sex typing. Psychological Review, 88(4), 354-364. doi:10.1037/0033-295X.88.4.354

Bem, S. L. (1982). Gender schema theory and self-schema theory compared: A comment on Markus, Crane, Bernstein, and Siladi's "self-schemas and gender". Journal of Personality and Social Psychology, 43(6), 1192-1194. doi: 10.1037/0022-3514.43.6.1192

Díaz-Aguado, M. J., Martínez Arias, R., Martín, G. y Toldos, M.P. (2001). Género, sexismo y expectativas sobre el futuro en el trabajo y en la pareja. En M. J. Díaz-Aguado y R. Martínez, (Coord.), La construcción de la igualdad y la prevención de la violencia contra la mujer desde la educación secundaria (pp. 235-285). Madrid. Instituto de la Mujer.

Finn, J. (1986). The relationship between sex role attitudes and attitudes supporting marital violence. Sex Roles, 14(5/6), 235-244. doi:10.1007/BF00287576

Gerber, G. L. (1989). Gender stereotypes: A new egalitarian couple emerges. En J. OffermanZuckerberg (Ed.), Gender in transition: A new frontier (pp. 47-66). New York: Plenum Medical Book Co.

Harter, S. (1990). Processes underlying adolescence self-concept formation. En R. Montemayor, G. R. Adams y T. P. Gullota (Eds.), From childhood to adolescence: A transitional period? (pp. 205239). Newbury Park, CA: Sage.

Helmreich, R. L., Spence, J. T., y Wilhelm, J. A. (1981). A psychometric analysis of the Personal Attributes Questionnaire. Sex Roles, 7(11), 1097-1108. doi: 10.1007/BF00287587

Klein, E., Campbell, J., Soler, E., y Ghez, M. (1997). Ending domestic violence: Changing public perceptions/halting the epidemic. Thousand Oaks: Sage Publications.

Markus, H., Crane, M., Bersntein, S. y Siladi, M. (1982). Self-schemas and gender. Journal of Personality and Social Psychology, 42(1), 38-50.

Pastorino, E., Dunham, R., Kidwell, J., Bacho, R., y Lamborn, S. (1997). Domain specific gender comparisons in identity development among college youth: Ideology and relationships. 
Adolescence, 32(127), 559-557.

Rodríguez, Y., Lameiras M., y Carrera, M. V. (2009). Análisis del espacio público versus privado en docentes no universitarios españoles. SUMMA Psicológica, 6(1), 15-24.

Rosenkrantz, P., Vogel, S., Bee, H., Broverman, I. y Broverman, D. M. (1968). Sex-role stereotypes and self-concepts in college students. Journal of Consulting and Clinical Psychology, 32(3), 287-295. doi:10.1037/h0025909

Siverio, M. A. y García, M. D. (2007). Autopercepción de adaptación tristeza en la adolescencia: La influencia de género. Anales de Psicología, 23(1), 41-48.

Spence, J. T. (1983). Comment on Lubinsky, Tellegan and Butcher's "maculinity, feminity and androgyny viewed and assessed as distinct concepts". Journal of Personaliy and Social Psychology, 44, 440-446. doi:10.1037/0022-3514.44.2.440

Spence, J. T. (1993). Gender-related traits and gender ideology: Evidence for a multifactorial theory. Journal of Personality and Social Psychology, 64(4), 624-635.

Spence, J., y Buckner, C. (2000). Instrumental and expressive traits, trait stereotypes and sexist attitude: what do they signify? Psychology of Women Quarterly, 24(1), 44-62. doi:10.1111/j.14716402.2000.tb01021.x

Spence, J. T., Helmreich, R. L., y Stapp. J. (1974). The Personal Attributes Questionnaire: A measure of sex role stereotypes and masculinity-femininity. JSAS Catalog of Selected Documents in Psychology, 4, 43-44.

Spence, J. T., Helmreich, W., y Stapp, J. (1975). Ratings of self and peers on sex role attributes and their relation to self-esteem and conceptions of masculinity and femininity. Journal of Personality and Social Psychology, 32(1), 29-39. doi:10.1037/h0076857

Straus, M. A., Kaufman, G., y Moore, D. W. (1997). Change in cultural norms approving marital violence from 1968 to 1994. En G. Kaufman y J. L. Jasinski (Eds.), Out of darkness: Contemporary perspectives on family violence (pp. 3-16). Thousand Oaks, CA: Sage.

Toldos M. P. (2002). Adolescencia, violencia y género (Tesis doctoral inédita). Facultad de Psicología. Universidad Complutense de Madrid.

Twenge, J. (1997). Changes in masculine and feminine traits over time: A meta-analysis. Sex Roles, 36(5/6), 305-325. doi:10.1007/BF02766650

Vergara, A. I., y Páez, D. (1993). Revisión teórico-metodológica de los instrumentos para la medición de la identidad de género. Revista de Psicología Social, 8(2), 132-152. 
